September 14, 2004

\title{
Helical rays in two-dimensional resonant wave conversion
}

\author{
Allan N. Kaufman \\ Lawrence Berkeley National Laboratory and \\ Physics Department, UC Berkeley, Berkeley, CA 94720 \\ Eugene R. Tracy \\ Physics Department \\ College of William and Mary \\ Williamsburg, VA 23187-8795 \\ Alain J. Brizard \\ Department of Chemistry and Physics \\ Saint Michael's College \\ Colchester, VT 05439
}

The process of resonant wave conversion (often called linear mode conversion) has traditionally been analyzed with a spatially one-dimensional slab model, for which the rays propagate in a two-dimensional phase space. However, it has recently been shown [E.R. Tracy and A.N. Kaufman, Phys. Rev. Lett. 91, 130402 (2003)] that multidimensional rays have a helical structure for conversion in two or more spatial dimensions (if their dispersion matrix is generic). In that case, a one-dimensional model is inadequate; a correct analysis requires two spatial dimensions and, thus, four-dimensional phase space. In this paper we show that a cold plasma model will exhibit ray helicity in conversion regions where the density and magnetic field gradients are significantly non-parallel. For illustration, we examine a model of the poloidal plane of a deuterium-tritium tokamak plasma, and identify such a region. In this region, characterized by a six-sector topology, rays in the sector for incident and reflected magnetosonic waves exhibit significant helicity. We introduce a "symmetricwedge" model, to develop a detailed analytic and numerical study of helical rays in this sector.

PACS Numbers: 52.35.Mw, 52.35.Bj, 42.25.Bs 


\section{INTRODUCTION}

Linear mode conversion occurs in a weakly nonuniform medium when two different wave types (i.e., waves with different polarizations and dispersion characterisitics) have the same frequency $\omega$ and nearly the same wave vector $\mathbf{k}$. This implies that, locally in $\mathbf{x}$, the two wave types have nearly equal phase velocities and one can resonantly excite the other. There is a large literature on linear mode conversion, because the phenomenon is known to arise in many different physical situations [1], with most theoretical studies of linear conversion using one-dimensional models (see, for example, Refs. [2], [3], and [4]). If the parameters describing the nonuniform medium (e.g., density and magnetic field) have gradients that are not parallel, however, a one-dimensional model is no longer valid, and a multidimensional theory of linear conversion must be developed.

Significant progress has been made toward this goal by building upon the seminal work of Friedland and Kaufman $[1,5,6]$. The approach uses Hamiltonian methods, which provide the most natural framework for formulating the linear conversion problem, because the resonance condition (for fixed $\omega$ ) requires both $\mathbf{x}$ and $\mathbf{k}$ to be nearly equal. Thus conversion occurs in a localized region of the ray phase space $(\mathbf{x}, \mathbf{k})$. Far from such regions, and away from caustics, Wentzel-Kramers-Brillouin (WKB) methods apply, and the wave equations for the two different waves can be solved via ray tracing. Within conversion regions, however, the WKB approximation breaks down, and a local $2 \times 2$ wave equation must be developed and solved, in order to connect the incoming and outgoing disturbances $[1,7]$. Once this is done, we find that, in the simplest multidimensional conversions, the process appears simply as a 'ray-splitting' event: an incoming ray splits into two outgoing rays, a transmitted and a converted ray. The amplitude and phase assignments for these outgoing rays are given by an $S$-matrix, whose entries are completely determined by an effective coupling constant [1]. In the most common conversion events, the rays will be confined to a two-dimensional osculating plane (in the ray phase space, not in physical space) and will exhibit an 'avoided-crossing' type of hyperbolic behavior in this two-dimensional plane in phase space. The magnitude of the effective coupling constant can be estimated from the ray geometry in this plane [8]. This has led to the development of a practical algorithm for ray tracing in tokamak geometry, including conversion between magnetosonic and ion hybrid waves; this is work in progress and will be reported elsewhere.

In addition to the most common type of linear conversion described above, it is possible in some cases for the ray geometry to be more complicated, with a breakdown of the approximation that rays are confined locally to an osculating plane. This occurs whenever the rays have helicity, a concept introduced in Ref. [9]. In helical conversion regions, the rays exhibit a combination of hyperbolic behavior in one two-dimensional subspace, and elliptical behavior in the complementary two-dimensional subspace. In Ref. [9], building upon methods of Littlejohn and Flynn [10, 11, 12], we examined ray helicity from an abstract point of view to emphasize the generality of the idea, and identified a new invariant that characterizes the rays in helical regions, a quantity we call the intrinsic helicity. In 
further work, we have developed a method of solution for the local wave fields in helical conversion, and have computed the WKB connection coefficients [13]. Here, we apply these ideas to a simple model of a tokamak plasma, and show that helical regions will occur.

The remainder of the paper is organized as follows. In Sec. II, the linearized magnetizedplasma response to a wave electric field of fixed frequency $\omega$ is treated by the standard coldplasma model [14], with density and magnetic field dependent on two spatial coordinates. In this paper, we investigate the application of ray helicity concepts to a simple twodimensional model, representing an idealization of a poloidal cross-section of a tokamak plasma, in which the poloidal magnetic field vanishes. Although nonzero poloidal magnetic fields would modify the details of the results, as will be discussed briefly in our summary and more fully in a separate paper, the essential qualitative aspects of our conclusions would be unchanged. In Sec. III, we focus our attention on a deuterium-tritium (DT) tokamak plasma, and study the regions of conversion of magnetosonic waves to ion-hybrid waves. In particular, the generic occurrence of six-sector regions, where the three curves for $L$-cutoff, $R$-cutoff, and ion-hybrid resonance all intersect, leads us to study the helical rays in the sector for incident and reflected magnetosonic waves. In Sec. IV, we then show that the essential aspects of the these rays can be captured by a very simple model that we call the "symmetric-wedge". Lastly, we summarize our work in Sec. V and discuss future plans.

\section{TWO-DIMENSIONAL COLD PLASMA MODEL}

\section{A. Cold-plasma Dispersion Matrix}

We take the background magnetic field $\mathbf{B}$ as unidirectional ( $z$-direction), with spatial variation $B(x, y)$. The unperturbed density (for each ion species $s$ ) is $n_{s}(x, y)$. (Twodimensionality appears through $\nabla B$ and $\nabla n$ being not parallel.) The wave electric field

$\mathbf{E}$ is purely perpendicular to $\mathbf{B}$, as is appropriate in the ion-gyrofrequency range. For this model, the wave equation for $\mathbf{E}$ is [14]:

$$
\mathrm{D}\left(\mathbf{k}_{\perp}=-i \nabla_{\perp}, \mathbf{x} ; k_{\|}, \omega\right) \cdot \mathbf{E}(\mathbf{x})=0
$$

where $\mathbf{x}=(x, y), \mathbf{k}_{\perp}=\left(k_{x}, k_{y}\right)$, the two-component wave electric field is $\mathbf{E}(\mathbf{x}) \exp \left(i k_{\|} z-\right.$ $i \omega t)$, and the $2 \times 2$ dispersion matrix $\mathrm{D}\left(\mathbf{k}_{\perp}, \mathbf{x} ; k_{\|}, \omega\right)$ is

$$
\left(\begin{array}{cc}
D_{x x} & D_{x y} \\
D_{x y}^{*} & D_{y y}
\end{array}\right)=\left(\begin{array}{cc}
\mathcal{S}-N_{\|}^{2}-N_{y}^{2} & -i \mathcal{D}+N_{x} N_{y} \\
i \mathcal{D}+N_{x} N_{y} & \mathcal{S}-N_{\|}^{2}-N_{x}^{2}
\end{array}\right),
$$

where $\mathbf{N}=c \mathbf{k} / \omega$ is the normalized wave vector, while $\mathcal{S} \equiv \frac{1}{2}(R+L)$ and $\mathcal{D} \equiv \frac{1}{2}(R-L)$, 
where

$$
\left(\begin{array}{l}
R \\
L
\end{array}\right)= \pm \sum_{s} \frac{\omega_{s}^{2}\left(n_{s}\right)}{\Omega_{s}(B)\left[\omega \pm \Omega_{s}(B)\right]} .
$$

Here, $\omega_{s}\left(n_{s}\right)=\left(4 \pi n_{s} e_{s}^{2} / m_{s}\right)^{\frac{1}{2}}$ and $\Omega_{s}(B)=e_{s} B / m_{s} c$ are the plasma frequency and the gyrofrequency of ion species $s$. We note that each element of the $2 \times 2$ matrix (2) is the sum of a function of $\mathbf{x}$ plus a function of $\mathbf{k}$; thus, the non-commutation of the operator $\mathbf{k}_{\perp}=-i \nabla_{\perp}$ with $\mathbf{x}$ presents no problem for the field equation (1). Note also that the dispersion matrix (2) generalizes the standard expression found in Ref. [14] by including two-dimensional effects (i.e., $k_{y} \neq 0$ ).

\section{B. Ray Hamiltonian Dynamics}

The ray representation of the wave field in four-dimensional ray phase space $\left(\mathbf{x}, \mathbf{k}_{\perp}\right)$ is governed by Hamilton's equations:

$$
\frac{d \mathbf{x}}{d \sigma}=-\frac{\partial H}{\partial \mathbf{k}_{\perp}} \text { and } \quad \frac{d \mathbf{k}_{\perp}}{d \sigma}=\frac{\partial H}{\partial \mathbf{x}}
$$

where $\sigma$ denotes the orbit parameter, and the ray Hamiltonian $H \equiv \operatorname{det}(\mathrm{D})$ is

$$
\begin{aligned}
H(\mathbf{x}, \mathbf{k}) & =R(\mathbf{x}) L(\mathbf{x})-\mathcal{S}(\mathbf{x})\left(2 N_{\|}^{2}+N_{\perp}^{2}\right)+N_{\|}^{2}\left(N_{\|}^{2}+N_{\perp}^{2}\right) \\
& =-\overline{\mathcal{S}}\left(\mathbf{x} ; N_{\|}\right) N_{\perp}^{2}+\bar{R}\left(\mathbf{x} ; N_{\|}\right) \bar{L}\left(\mathbf{x} ; N_{\|}\right),
\end{aligned}
$$

where $N_{\perp}^{2}=N_{x}^{2}+N_{y}^{2}$ and $(\bar{R}, \bar{L}, \overline{\mathcal{S}})=\left(R-N_{\|}^{2}, L-N_{\|}^{2}, \mathcal{S}-N_{\|}^{2}\right)$. The rays lie on the three-dimensional manifold $H\left(\mathbf{x}, \mathbf{k}_{\perp} ; k_{\|}\right)=0$, so that

$$
N_{\perp}^{2}\left(\mathbf{x} ; N_{\|}\right)=\frac{\bar{R} \bar{L}}{\overline{\mathcal{S}}} .
$$

The rays are thus bounded by the zero-curves of $\bar{R}$ and $\bar{L}$ (denoted as "cutoffs") and by the zero-curve of $\overline{\mathcal{S}}$ (denoted as "resonance"); these curves are displayed in Figure 4.

\section{Helicity Invariants}

Following the ideas of Littlejohn and Flynn [12], we express the dispersion matrix (2) in the Pauli-matrix basis, so that its elements compose the Lorentz-like four-vector $D^{\mu}$ (denoted $B^{\mu}$ in Ref. [9]):

$$
\left(\begin{array}{c}
D^{0} \\
D^{1} \\
D^{2} \\
D^{3}
\end{array}\right)=\left(\begin{array}{c}
\frac{1}{2} \operatorname{Tr}(\mathrm{D}) \\
\operatorname{Re}\left(D_{x y}\right) \\
-\operatorname{Im}\left(D_{x y}\right) \\
\frac{1}{2}\left(D_{x x}-D_{y y}\right)
\end{array}\right)=\left(\begin{array}{c}
\mathcal{S}(\mathbf{x})-N_{\|}^{2}-\frac{1}{2} N_{\perp}^{2} \\
N_{x} N_{y} \\
\mathcal{D}(\mathbf{x}) \\
\frac{1}{2}\left(N_{x}^{2}-N_{y}^{2}\right)
\end{array}\right)
$$


and, thus, $\operatorname{det}(\mathrm{D})=D^{\mu} \eta_{\mu \nu} D^{\nu}$, using the Minkowski metric $\eta_{\mu \nu}=\operatorname{diag}(1,-1,-1,-1)$ with summation over repeated indices. Along a ray, the dispersion four-vector evolves as [9]: $d D^{\mu} / d \sigma=2 \Omega^{\mu \nu} D_{\nu}$, where the Lorentz-like four-tensor

$$
\Omega^{\mu \nu} \equiv\left\{D^{\mu}, D^{\nu}\right\}
$$

is defined in terms of the Poisson brackets of the components of the four-vector (7). Constant congruence transformations of $\mathrm{D}$ (with unit determinant), which linearly combine the components of the wave field $\mathbf{E}$ (i.e., change the polarization basis), induce Lorentz transformations of the four-tensor (8). We define genericity of the dispersion matrix D as the nonvanishing of the determinant of the tensor $\Omega^{\mu \nu}$. For our model, it is straightforward to evaluate $\operatorname{det}(\boldsymbol{\Omega})$ from Eqs. (7)-(8) as

$$
\operatorname{det}(\boldsymbol{\Omega})=\left(\Lambda_{\perp}^{2} \widehat{\mathbf{z}} \cdot \nabla \mathcal{S} \times \nabla \mathcal{D}\right)^{2}
$$

where $\boldsymbol{\Lambda}_{\perp}=c \mathbf{N}_{\perp} / \omega$. Since $\mathcal{S}$ and $\mathcal{D}$ depend on position $\mathbf{x}$ through $B$ and $n$, the nonvanishing of $\operatorname{det}(\boldsymbol{\Omega})$ follows from $\nabla B$ and $\nabla n$ being nonparallel.

By analogy to the electromagnetic four-tensor representing the three-vector electric and magnetic fields, one can express the antisymmetric tensor $\Omega$ in terms of the two threevectors $\gamma$ and $\boldsymbol{\omega}$ :

$$
\gamma_{i}=\Omega_{0 i} \quad \text { and } \quad \omega^{i}=\frac{\epsilon^{i j k}}{2} \Omega_{j k} \quad(\text { with } i, j, k=1,2,3),
$$

so that $\operatorname{det}(\boldsymbol{\Omega})=(\boldsymbol{\gamma} \cdot \boldsymbol{\omega})^{2}$. As explained more fully elsewhere [9], the three-vector $\boldsymbol{\gamma}$ defines both the direction (in $D^{\mu}$-space) and magnitude of the locally hyperbolic motion of the ray, while $\boldsymbol{\omega}$ defines both the axis of rotation (again in $D^{\mu}$-space) and the local rate of rotation of the ray. Based on the four-vector (7) and the definition of the four-tensor (8), the vectors $\boldsymbol{\gamma}$ and $\boldsymbol{\omega}$ defined in Eq. (10) are

$$
\boldsymbol{\gamma}=\left(\begin{array}{c}
\Lambda_{x} \partial_{y} \mathcal{S}+\Lambda_{y} \partial_{x} \mathcal{S} \\
\Lambda_{x} \partial_{x} \mathcal{D}+\Lambda_{y} \partial_{y} \mathcal{D} \\
\Lambda_{x} \partial_{x} \mathcal{S}-\Lambda_{y} \partial_{y} \mathcal{S}
\end{array}\right) \quad \text { and } \quad \boldsymbol{\omega}=\left(\begin{array}{c}
-\Lambda_{x} \partial_{x} \mathcal{D}+\Lambda_{y} \partial_{y} \mathcal{D} \\
0 \\
\Lambda_{x} \partial_{y} \mathcal{D}+\Lambda_{y} \partial_{x} \mathcal{D}
\end{array}\right)
$$

thus $|\gamma|^{2}=\Lambda_{\perp}^{2}|\nabla \mathcal{S}|^{2}+\left(\Lambda_{\perp} \cdot \nabla \mathcal{D}\right)^{2},|\boldsymbol{\omega}|^{2}=\Lambda_{\perp}^{2}|\nabla \mathcal{D}|^{2}$, and $\boldsymbol{\gamma} \cdot \boldsymbol{\omega}=\Lambda_{\perp}^{2} \widehat{z} \cdot \nabla \mathcal{S} \times \nabla \mathcal{D}$. The congruence/Lorentz invariants are thus

$$
\left.\begin{array}{rl}
\boldsymbol{\gamma} \cdot \boldsymbol{\omega} & =-\frac{1}{2} \Lambda_{\perp}^{2} \widehat{\mathbf{z}} \cdot \nabla R \times \nabla L \\
|\boldsymbol{\omega}|^{2}-|\gamma|^{2} & =-\Lambda_{\perp}^{2} \nabla R \cdot \nabla L-\left(\boldsymbol{\Lambda}_{\perp} \cdot \nabla \mathcal{D}\right)^{2}
\end{array}\right\} .
$$

Allowing for scaling transformations (nonunit-determinant congruence transformations) leads to an equation for a single quantity, called the helicity invariant, that is invariant under all constant congruence transformations:

$$
K \equiv \frac{|\boldsymbol{\omega}|^{2}-|\gamma|^{2}}{\gamma \cdot \omega}
$$


From Eqs. (11) and (12), the helicity invariant (13) becomes

$$
K=\frac{\nabla R \cdot \nabla L+\left(\widehat{\mathrm{N}}_{\perp} \cdot \nabla \mathcal{D}\right)^{2}}{\frac{1}{2} \hat{\mathrm{z}} \cdot(\nabla R \times \nabla L)} .
$$

This nonzero result shows that a proper treatment of two-dimensional conversion requires the explicit consideration of helicity. Note that invariance under congruence should not be confused with dynamical invariance as $K$ varies significantly along a ray (see Figure 13 ).

The vectors $\boldsymbol{\gamma}$ and $\boldsymbol{\omega}$ can always be made parallel or antiparallel via a Lorentz transformation - implying there is a congruence transformation that makes it so. Denote the values of these vectors in that special frame as $\gamma_{0}$ and $\boldsymbol{\omega}_{0}$. In this special frame, we have $\boldsymbol{\gamma}_{0} \cdot \boldsymbol{\omega}_{0}=\epsilon\left|\gamma_{0}\right|\left|\boldsymbol{\omega}_{0}\right|$, where the sign $\epsilon= \pm 1$ reflects the handedness of the helical orbit. From Eq. (13), thus, we can write $K \equiv \kappa-\kappa^{-1}$, where we define the ray helicity as $\kappa \equiv \epsilon\left|\boldsymbol{\omega}_{0}\right| /\left|\boldsymbol{\gamma}_{0}\right|$. In the next two Sections, we consider a specific model, and numerically compute the rays to display this helical effect.

\section{HELICAL RAYS IN A DEUTERIUM-TRITIUM TOKAMAK PLASMA}

We consider a neutral plasma with equal densities of deuterium and tritium throughout $\left(n_{D}=n_{T}\right)$. The minor radius of the tokamak plasma is taken to be $a=100$ $\mathrm{cm}$, and the densities are assumed to be radially symmetric with a Gaussian profile: $n(r)=n_{0} \exp \left(-r^{2} / a^{2}\right)$, with $n_{0}=1.2 \times 10^{14} \mathrm{~cm}^{-3}$. The magnetic field strength is taken as $B(x)=B_{0}\left(1+x / L_{B}\right)^{-1}$, with $B_{0}=5.85 \mathrm{~T}$ and $L_{B}=10^{3} \mathrm{~cm}$. The wave frequency is chosen to be of the order of the ion-hybrid ( $\mathrm{IH})$ resonance frequency, while parameters are chosen so that neither gyroresonance lies within the plasma.

\section{A. Spatial Functions $R(\mathbf{x})$ and $L(\mathbf{x})$}

First, we consider the nature of the functions $R(\mathbf{x})$ and $L(\mathbf{x})$ given by Eq. (3) and shown in Figures 1 and 2. Figure 1 shows that $R(\mathbf{x})$ is peaked near the magnetic axis and falls off monotonically toward the plasma edge near $r \sim a$ (i.e., $R$ varies essentially like $n$ ). This function is zero only at the plasma edge where the density goes to zero. Figure 2 shows that the function $L(\mathbf{x})$ is zero at the plasma edge and at the $L$-cutoff of the magnetosonic waves (for $N_{\|}=0$ ). In the interior of the plasma, $L(\mathbf{x})$ is nearly linear in $x$ and constant in $y$ (i.e., $L$ varies strongly with $B$ and only weakly with $n$ in the interior of the plasma). As these Figures show, the ion-hybrid resonance is well approximated by a line $x=x_{I H}$, 


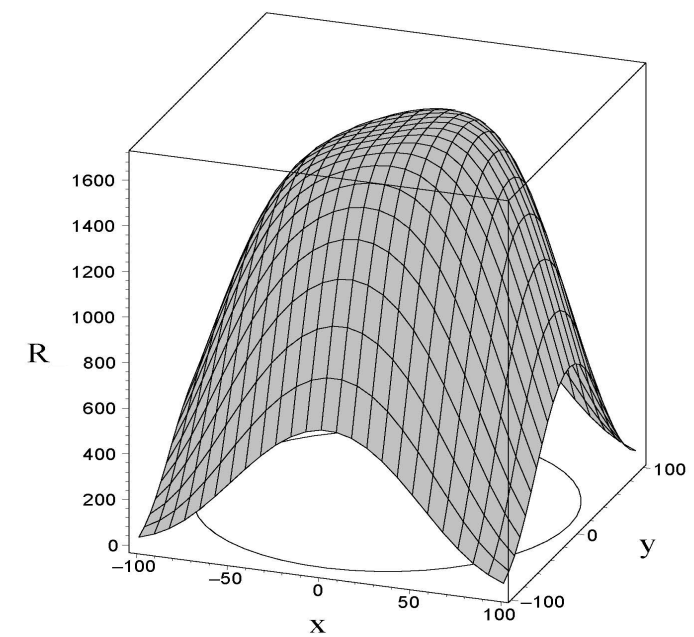

Figure 1: Plot of $R(x, y)$ for a cold D-T plasma; note that $R$ varies essentially like $n$.

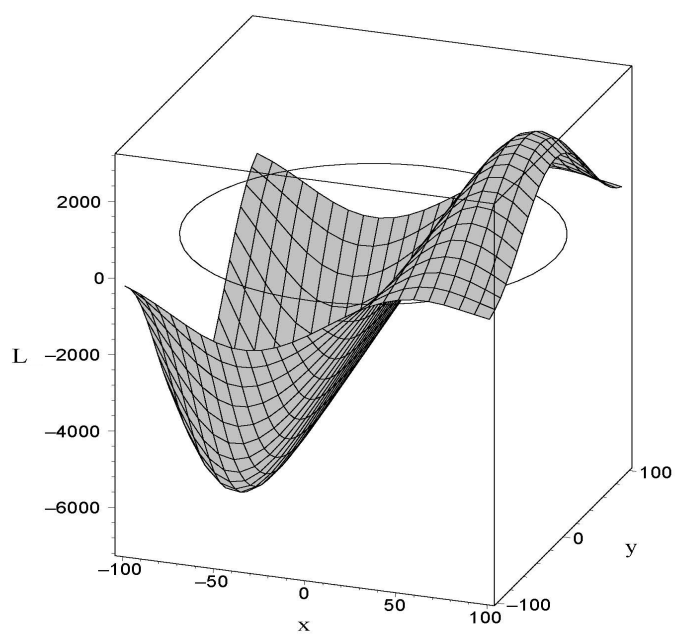

Figure 2: Plot of $L(x, y)$ for a cold D-T plasma; note that $L$ varies strongly with $B$ and only weakly with $n$ so that $\nabla L$ is in the $x$-direction in the plasma interior. 
while the position of the resonance depends upon the wave frequency $\omega$.

\section{B. Two-dimensional Dispersion Plots}

Setting $y=0$ and $N_{y}=0$, Figure 3 shows the zero locus of the dispersion function (5) on the tokamak midplane: $H\left(x, k_{x} ; y=0=k_{y}\right)=0$ for $N_{\|}=20$ (solid) and $N_{\|}=10$ (dashed). Figure 3 suggests that the dispersion surface has two distinct sheets, one on the high-field side and one on the low-field side of the ion-hybrid resonance layer, as well as two conversion regions (one for $N_{x}>0$ and one for $N_{x}<0$ ), with tunneling in each. Notice also the confinement of rays at the plasma edge, which suggests that magnetosonic (MS) rays launched at the plasma edge on the low-field side circulate in a clockwise sense and tunnel through to the other sheet on each pass through the ion-hybrid ( $\mathrm{IH}$ ) resonance region. It is also important to note that the dispersion surfaces are sensitively dependent upon the value of $N_{\|}^{2}$, and that increasing $N_{\|}^{2}$ brings the two sheets of the dispersion surface closer together (implying larger transmission). Because of the symmetry of the dispersion function under reflection through the mid-plane $(y \rightarrow-y)$, any ray launched in the midplane (with $y=0=N_{y}$ ) will remain on it, along with the rays that it tunnels and/or converts to.

The local dispersion function for WKB-type waves is the determinant of the dispersion matrix $H=\operatorname{det}(\mathrm{D})$, which, from Eq. (5), can be written as the sum of a "kinetic" term and a "potential" term

$$
0=H\left(\mathbf{x}, \mathbf{k}_{\perp}\right) \equiv-2 \overline{\mathcal{S}}(x, y)\left[\frac{N_{\perp}^{2}}{2}+U(x, y)\right]
$$

where the effective potential is

$$
U(x, y) \equiv-\frac{\bar{R}(x, y) \bar{L}(x, y)}{2 \overline{\mathcal{S}}(x, y)} .
$$

Thus, ray propagation $\left(N_{\perp}^{2}>0\right)$ requires that $U(x, y)<0$. This requirement can be satisfied in three separate regions of the $(x, y)$-plane: in region I, where $\bar{R}, \bar{L}$, and $\overline{\mathcal{S}}$ are all positive; in region II, where $\bar{L}$ and $\overline{\mathcal{S}}$ are both negative but $\bar{R}$ is positive; and in region III, where $\bar{R}$ and $\overline{\mathcal{S}}$ are both negative but $\bar{L}$ is positive. As we see in Figure 4 , the three propagating regions are separated by three evanescent regions in which $U(x, y)>0$; all six regions meet at the intersection points where $\bar{L}(x, y), \bar{R}(x, y)$, and $\overline{\mathcal{S}}(x, y)$ vanish.

We further investigate the two-dimensional structure in the vicinity of the intersection points in Figures 4(a)-(c). In Figure 4(a), we plot the zero- and infinity-loci of the potential $U(x, y)$, where $\bar{R}=0=\bar{L}$ (dark curves) and $\overline{\mathcal{S}}=0$ (light curve), respectively, for $N_{\|}=20$. The important features of the zero locus of $U$ in Figure 4(a) are the circle, which shows the outermost radial excursion of magnetosonic rays (i.e., the radial turning points), and the vertical line representing the cutoff caustic of the magnetosonic rays. 


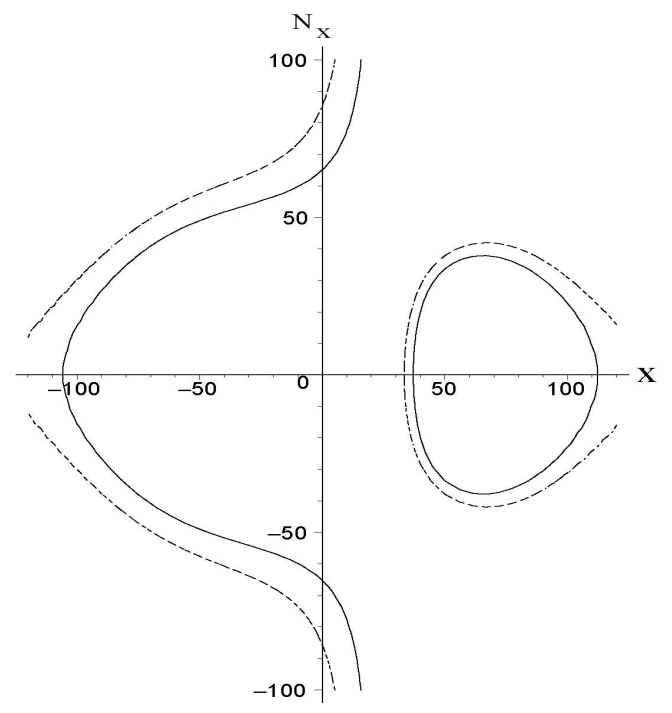

Figure 3: Plot of $H\left(x, k_{x} ; y=0=k_{y}\right)=0$, for $N_{\|}=20$ (solid) and $N_{\|}=10$ (dashed). The plot suggests that the dispersion surface has two distinct sheets, one on the high-field side and one on the low-field side of the ion-hybrid resonance layer, as well as two conversion regions (one for $N_{x}>0$ and one for $N_{x}<0$ ), with tunneling in each.
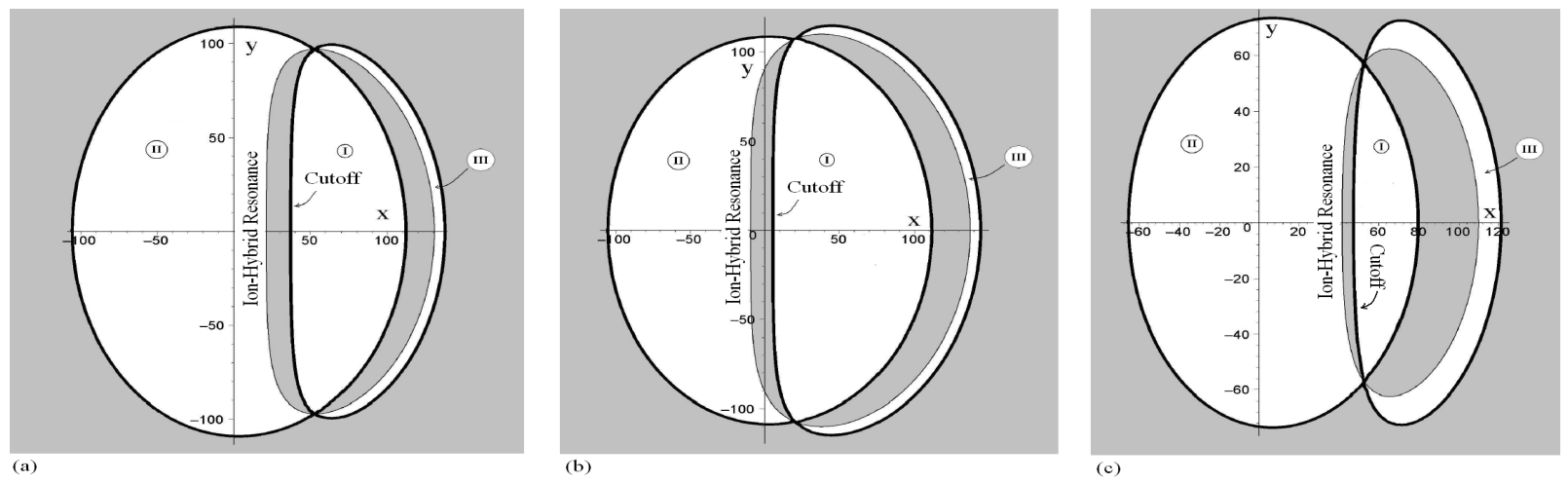

Figure 4: Plots of cutoffs $\bar{R}(\mathbf{x})=0$ and $\bar{L}(x, y)=0$ (dark curves) and the ion-hybrid resonance $\overline{\mathcal{S}}(\mathbf{x})=0$ (light curve) for (a) $N_{\|}(=20)$ and frequency $\omega$, (b) same $N_{\|}$and higher frequency $\omega$, (c) same frequency $\omega$ and higher $N_{\|}$; the regions of ray propagation $(U<0)$ are unshaded while the evanescent regions $(U>0)$ are shaded. The triple intersections, where all three curves cross, produce six sectors (see Figure 5), where two-dimensionality is paramount. 
(a)

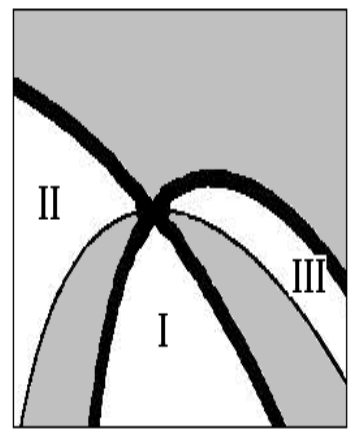

(b)

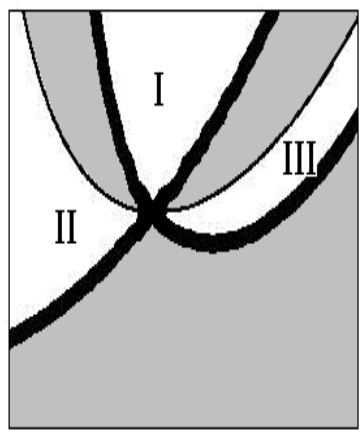

Figure 5: Two-dimensional structures in (a) the upper wedge region and (b) the lower wedge region, determined by the curves $\bar{R}(x, y)=0, \bar{L}(x, y)=0$, and $\overline{\mathcal{S}}(x, y)=0$, showing the three propagating regions (I, II, III) and the three evanescent regions (shaded).

The light vertical line associated with $\overline{\mathcal{S}}(x, y)=0$ (the infinity-locus of $U$ ) shows the ion-hybrid resonance line located between the magnetic axis and the cutoff line. Figure 4(a) also shows the three propagating regions (I, II, III) and the three evanescent regions (shaded). Notice that the incident and reflected magnetosonic rays propagate in region I, while transmitted and converted rays propagate in region II; both regions II and III will be the subject of future investigation. Figures 4(b)-(c) demonstrate the genericity of the six-sector topology by showing, respectively, that either an increase in wave frequency $\omega$ or an increase in $N_{\|}$only changes the location of the intersection points. We remark that the two-dimensional structure of each wedge region (see Figure 5) motivates the introduction of a simple symmetric-wedge model in Sec. IV used to investigate the nature of helical rays in region $\mathrm{I}$.

For $N_{\|}^{2} \neq 0$, the intersection points (where all six regions meet) occur in the interior of the plasma. At each intersection point, the two sheets of the dispersion surface touch and rays in the vicinity of a conversion point exhibit a very interesting and complex helical behavior that is necessarily absent from one-dimensional models. Because the two sheets of the dispersion surface touch at these points, there should be significant transmission of wave energy from one sheet to the other in the vicinity of these points.

In Figure 6, the contour plots of $\bar{R}(\mathbf{x})$, which scales with the plasma density profile (i.e., it is large and positive inside the dark circle and negative outside), show that the $\bar{R}$-profile (which is closely related to the effective coupling coefficient) passes through zero in each wedge region, which leads to significant transmission. The possibility of such essentially multidimensional wedge regions has been largely overlooked in prior work on mode conversion [15]. Although the wedge regions are spatially isolated, they can potentially produce 


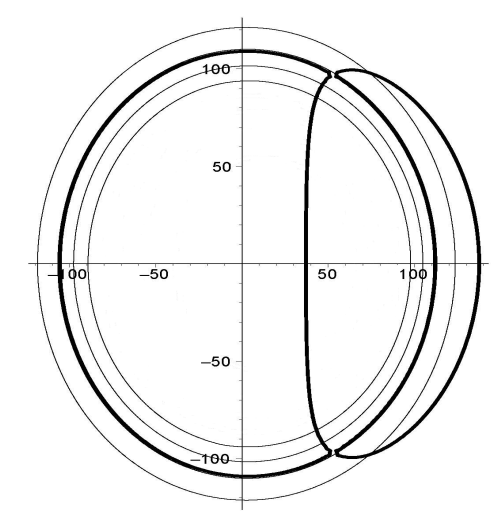

Figure 6: Contour plots of $\bar{R}(x, y)$ (associated with the effective coupling constant) and the zero locus of $H\left(x, y, k_{x}=0, k_{y}=0\right)=0$ (dark curves).

a significant modification to the global wave field.

\section{Three-dimensional Dispersion Plot}

Lastly, we wish to gain a more global perspective on these issues. However, this is difficult because of the four-dimensionality of ray phase space. It is clear that two-dimensional slices of the dispersion surfaces (i.e., plots of the zero locus of $H=0$ confined to various two-dimensional planes) convey limited information. The condition $H=0$ defines a threedimensional surface embedded in the four-dimensional phase space. We can gain more insight by looking at the dispersion surface in various three-dimensional slices. Figure 7 shows the dispersion surface confined to $N_{y}=0$ (for $N_{\|}=20$ ) in the three-dimensional subspace $\left(x, y, N_{x}\right)$. It is important to note that this still conveys only limited information because rays lie on this surface only when $N_{y}=0$; hence, it would be useful to look at a sequence of such figures for different values of $N_{y}$.

Although Figure 7 needs to be interpreted with care, it reveals several important features: note that it looks roughly like an ellipsoid intersected by a cylinder. This can be understood as follows: if we turn off the effects of the ion-hybrid resonance, the magnetosonic wave would have a dispersion surface that has the shape of an ellipsoid in phase space, because it is confined within the plasma in the $(x, y)$-plane, and it is also confined in the $\left(N_{x}, N_{y}\right)$-plane because it is quadratic in those variables. The ion-hybrid resonance defines a three-dimensional cylindrical quasiplane in phase space. Including the interaction between the magnetosonic wave and the ion-hybrid resonance causes these two intersecting 


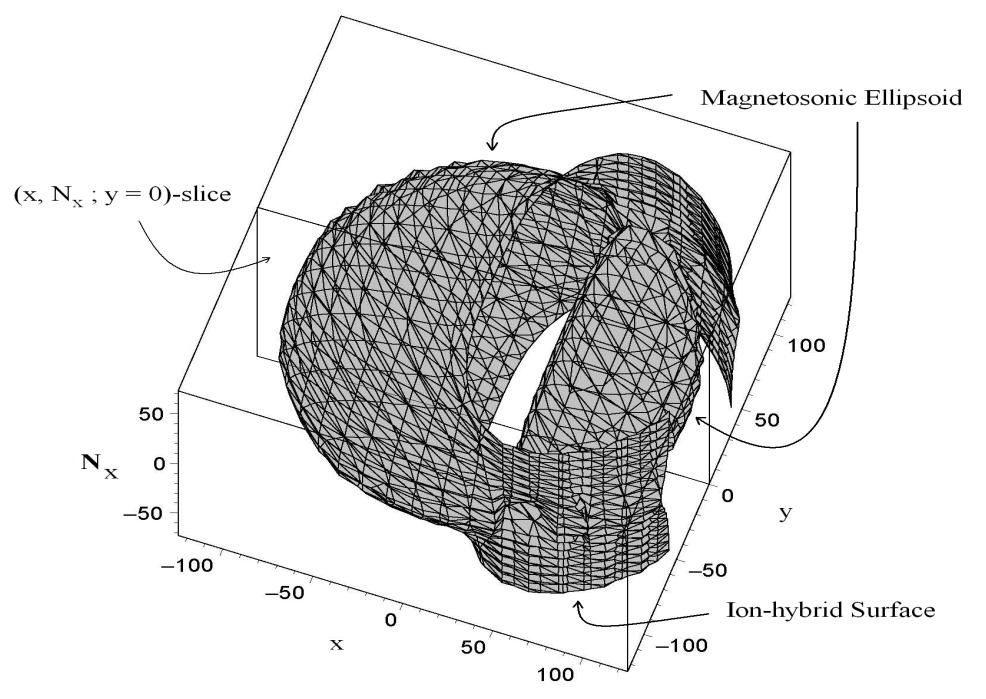

Figure 7: Plot of $H\left(x, y, k_{x}, k_{y}=0\right)=0$; the two-dimensional $\left(x, N_{x} ; y=0\right)$-slice can be seen in Figure 3 for $N_{\|}=20$. 
surfaces to reconnect in the manner shown in Figure 7.

Note that the piece of the dispersion surface (which refers to the entire set of points where $H=0$ ) on the low-field side of the ion-hybrid resonance is localized in phase space, while the piece on the high-field side extends to infinity. This is important because it means that, although the rays are confined spatially within the plasma, rays can escape to large values in $N_{x}$. Physically, this means that the wave develops small-scale spatial structure, whose amplitude will eventually damp by particle resonance.

\section{SYMMETRIC-WEDGE MODEL}

Referring to Eq. (14) for the helicity invariant $K$, we note its simple dependence on the ray direction $\widehat{N}$. For the case that $\nabla R \cdot \nabla L<0$ (see Figure 8), the numerator of $K$ is extremized by taking $\widehat{N}$ orthogonal to $\nabla \mathcal{D}$, whence we obtain $|K|_{\max }=2 \cot \alpha$, where $\alpha$ is the (acute) angle between $R$ and $L$ contours. The trigonometric identity $2 \cot \alpha=$ $\cot (\alpha / 2)-\tan (\alpha / 2)$ then yields the maximum intrinsic ray helicity

$$
|\kappa|_{\max }=\cot (\alpha / 2) .
$$

Remarkably, this result is independent of the ratio $|\nabla R| /|\nabla L|$, which motivates us to pursue the simplest model $|\nabla R|=|\nabla L|$.

\section{A. Wedge Hamiltonian}

In the neighborhood of each intersection point (e.g., Figure 5), where $\bar{R}$ and $\bar{L}$ both vanish, we may approximate $\bar{R}$ and $\bar{L}$ by the leading terms of their Taylor expansions:

$$
\left.\begin{array}{l}
\bar{R}(\xi, \eta)=\beta(b \xi-\eta) \\
\bar{L}(\xi, \eta)=\beta(b \xi+\eta)
\end{array}\right\},
$$

where the new coordinates $(\xi, \eta)$ have been chosen to exhibit the bilateral symmetry of the potential $U(\xi, \eta)$ given by Eq. (16), so that the origin corresponds to the location where $\bar{R}=0=\bar{L}$. In Eq. (18), the symmetric-wedge model parameters are: $b=\tan (\alpha / 2)$, where $\alpha$ is the wedge angle between the edges $\bar{R}=0$ and $\bar{L}=0$ (see Figure 8), and $\beta$ is a constant (so that $|\nabla \bar{R}|=\beta \sqrt{b^{2}+1}=|\nabla \bar{L}|$ ). In the symmetric-wedge model (18), we also have $\overline{\mathcal{S}}=\beta b \xi$ and $\overline{\mathcal{D}}=-\beta \eta$, so that Eq. (14) becomes

$$
K=\tan (\alpha / 2)-\left(N_{\xi} / N_{\perp}\right)^{2} \cot (\alpha / 2),
$$

which is indeed maximized when $N_{\eta}=0$ (with $\left.\gamma=-\boldsymbol{\omega} \tan \alpha / 2\right)$. In Figure 8, we show that orbit propagation is allowed in three regions (labeled I, II, and III) in which $U(\xi, \eta)<0$, while orbit propagation is forbidden in three regions (shaded gray) in which $U(\xi, \eta)>0$. Note that increasing the parameter $b$ increases region I at the expense of regions II and III. 


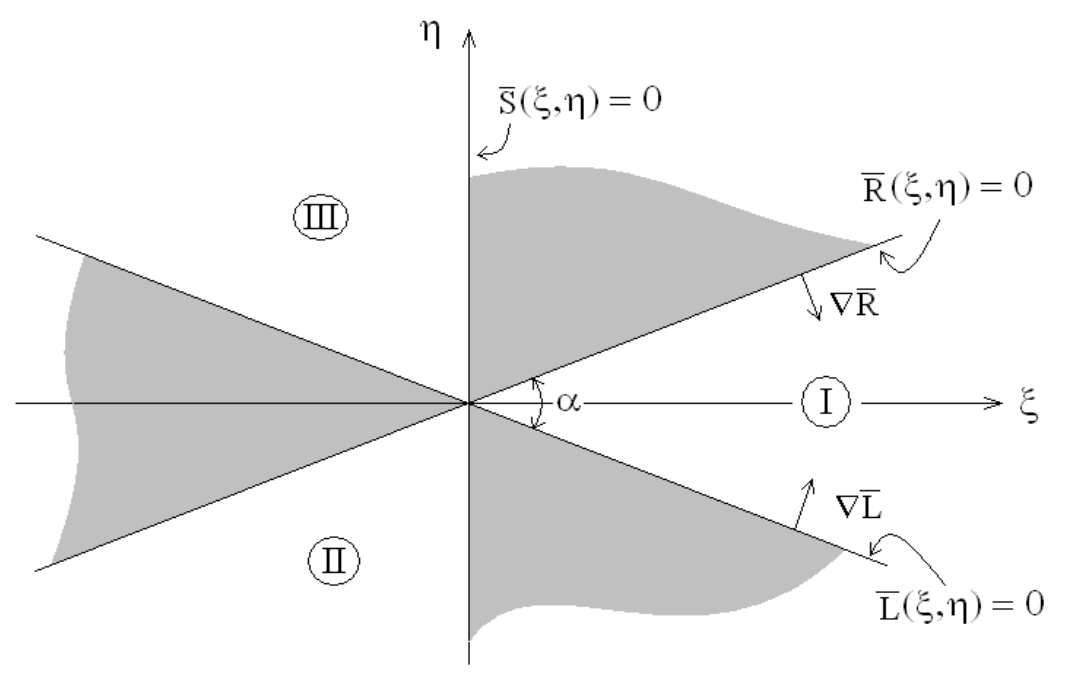

Figure 8: Propagating (unshaded) and evanescent (shaded) sectors for the symmetricwedge model; note that the angles of the propagating regions add up to $\pi$. A magnetosonic wave enters from the right in region I, its rays undergoing helical orbits in this region (see Figures 10, 11, and 12). The wave tunnels to regions II and III, but this phenomenon is absent from the ray representation studied in this paper.

Let us reparametrize $\sigma \rightarrow \tau$ by introducing a new orbit parameter $\tau$ and rescale the wavevector $\mathbf{k}$ as

$$
d \tau \equiv \frac{c}{\omega} \sqrt{\beta} d \sigma \quad \text { and } \quad\left(\frac{c k_{\xi}}{\omega \sqrt{\beta}}, \frac{c k_{\eta}}{\omega \sqrt{\beta}}\right) \equiv\left(p_{\xi}, p_{\eta}\right),
$$

so that the ray Hamiltonian (15) becomes

$$
H^{\prime}\left(\xi, p_{\xi}, \eta, p_{\eta}\right)=\frac{1}{2}\left(p_{\xi}^{2}+p_{\eta}^{2}\right)+U^{\prime}(\xi, \eta)
$$

with the effective potential

$$
U^{\prime}(\xi, \eta) \equiv \beta^{-1} U(\xi, \eta)=\frac{1}{2}\left(-b \xi+\frac{\eta^{2}}{b \xi}\right) .
$$

The three propagation regions are now defined as: (I) $\xi>0$ and $-b \xi<\eta<b \xi$, (II) $\xi<0$ and $\eta<b \xi<0$, and (III) $\xi<0$ and $\eta>-b \xi>0$.

Figure 9 shows a plot of the regions where $U^{\prime}(\xi, \eta)<0$; here, we note that region I is characterized by a shallow potential while regions II and III (barely visible) are characterized by very steep potentials that become infinite as $\xi \rightarrow 0^{-}$. Note that, because $H^{\prime}=0$, the magnitude of the ray "momentum" $|\mathbf{p}|=\sqrt{-2 U^{\prime}(\xi, \eta)}$ increases with the depth of the 


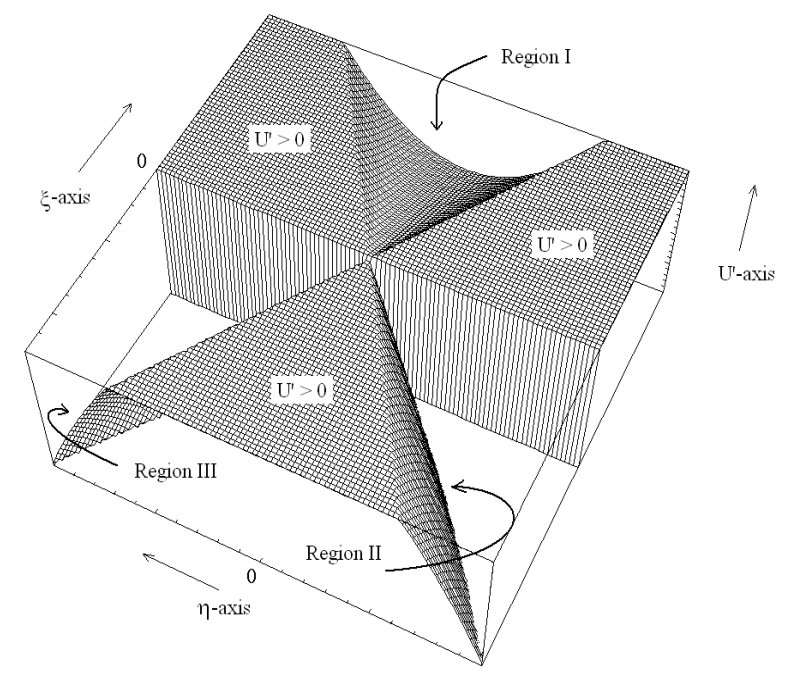

Figure 9: Plot of potential function $U^{\prime}(\xi, \eta)<0$.

potential $U^{\prime}(\xi, \eta)$. The shape of $U^{\prime}(\xi, \eta)$ in region I implies the existence of an accelerating field in the positive- $\xi$ direction and a confining potential in the $\eta$-direction leading to oscillatory $\eta$-motion. We now investigate rays in region $\mathrm{I}$, and leave the investigation of rays in regions II and III to future work.

\section{B. Helical Rays in Region I}

Using the wedge Hamiltonian (20), the dynamics is now described by the Hamilton equations:

$$
\begin{array}{ll}
\dot{\xi}(\tau)=p_{\xi}(\tau), & \dot{p}_{\xi}(\tau)=\frac{1}{2}\left(b+\frac{\eta^{2}(\tau)}{b \xi^{2}(\tau)}\right) \\
\dot{\eta}(\tau)=p_{\eta}(\tau), & \dot{p}_{\eta}(\tau)=-\frac{\eta(\tau)}{b \xi(\tau)}
\end{array}
$$

Figure 10, which shows the projections of Hamiltonian rays on the $(\xi, \eta)$-plane, deserves a few comments. First, note the oscillatory behavior along the $\eta$-direction, while the $\xi$-motion displays the influence of a retarding force. Second, we note that, upon closer inspection, although rays come close to the edges of the wedge, they do not touch them (see inset). Third, we note that the reflection of the rays at the upper and lower edges lead to energy tunneling into regions III and II, respectively. Lastly, we note that the ray (D), with the smallest initial value $\eta_{0}$, reaches farthest into the wedge, since the strength of the retarding force, given by the second equation in Eq. (22), decreases with $\eta$ at fixed values of $b$ and $\xi$. Figures 11 and 12 show various two-dimensional and three-dimensional views of ray D 


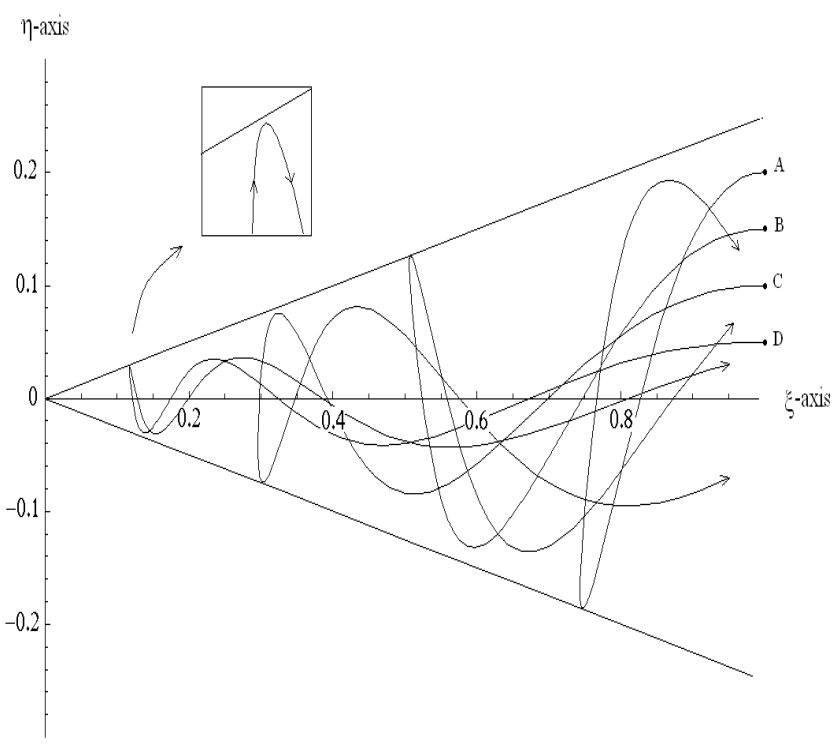

Figure 10: Rays in Region I for $b=0.25, \xi_{0}=1, p_{\eta 0}=0, p_{\xi 0}=-\sqrt{2\left|U^{\prime}\left(\xi_{0}, \eta_{0}\right)\right|}$, and various initial conditions in $\eta: \eta_{0}=0.2(A), 0.15(B), 0.1(C), 0.05(D)$. The inset shows that, although rays come close to the edges of the wedge, they do not touch them.

shown in Figure 10, respectively, where the initial position is marked by a dot and the direction of evolution is indicated by arrows. In Figures 11(a)-(b), the $\xi$-motion shows the effects of the retarding force, while in Figures 11(c)-(d), the oscillatory $\eta$-motion is clearly seen. In Figures 12(a)-(b), we can see the complex three-dimensional nature of the helical rays in region I as the magnetosonic wave gets reflected by the edges of the wedge.

For the central ray $\left[\eta(\tau)=0=p_{\eta}(\tau)\right]$, the equations of motion (22) yield $\ddot{\xi}=b / 2$, with solution $\xi(\tau)=b \tau^{2} / 4$. For infinitesimal $\left(\eta, p_{\eta}\right)$, the $\eta$-motion is essentially that of a harmonic oscillator (see Figure 11(d)), with a $\xi$-dependent frequency $\omega_{\eta}^{2}=1 /[b \xi(\tau)]$, so that $\omega_{\eta}(\tau)=2 /(b \tau)$. Defining the local exponentiation rate $\gamma(\tau)$ of the $\xi$-motion by $\gamma_{\xi}(\tau)=\dot{\xi}(\tau) / \xi(\tau)$, we have $\gamma_{\xi}(\tau)=2 / \tau$. We then can define the heuristic ray helicity by $\kappa(\tau) \equiv \omega_{\eta}(\tau) / \gamma_{\xi}(\tau)$, and obtain $\kappa(\tau)=1 / b=\cot (\alpha / 2)$, in exact agreement with the formal result (17). Figure 13 shows that the helicity $K(\tau)$, rewritten from Eq. (19) as $K(\tau)=b-\left(p_{\xi} / p_{\perp}\right)^{2} b^{-1}$, reaches its maximum (negative) value $K_{\max }$ when the oscillatory $\eta$-motion reaches its turning points (where $p_{\eta}=0$ and $p_{\xi} / p_{\perp}=1$ ).

\section{Wedge Adiabatic Invariant}

Looking at the ray projection onto the $\left(\eta, p_{\eta}\right)$ shown in Figure $11(\mathrm{~d})$, one can readily notice the harmonic oscillation of the $\eta$-motion and the adiabatic invariance of the enclosed area. For an acute wedge angle $(\alpha<\pi / 2)$, the $\eta$-oscillation frequency $\omega_{\eta}$ exceeds the $\xi$ - 
(a) $p_{\xi}$-axis

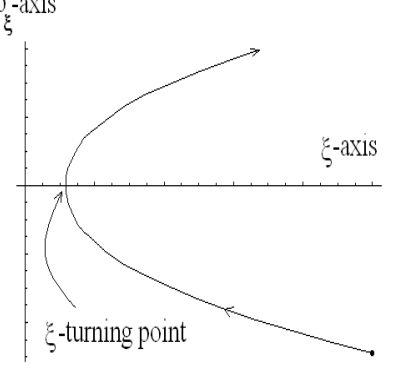

(c)

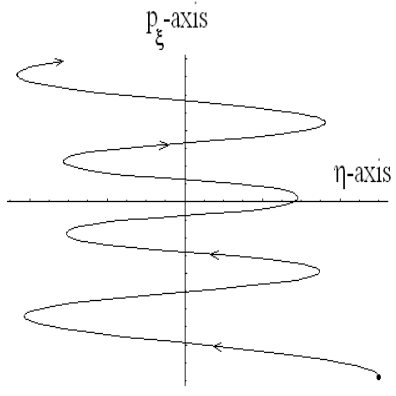

(b) $p_{\text {- }}$-xis

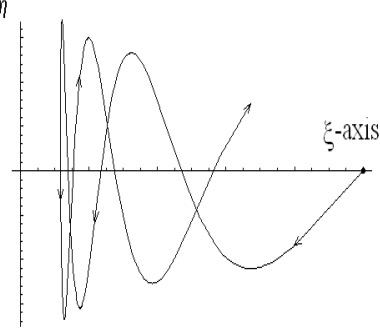

(d)

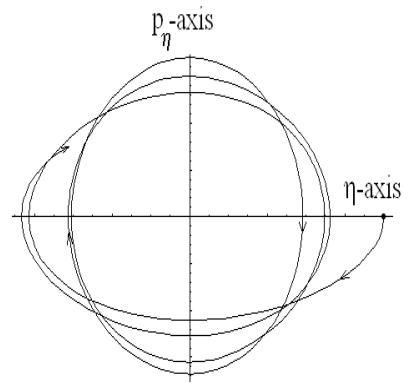

Figure 11: Two-dimensional views of ray D of Figure 10.

(a)

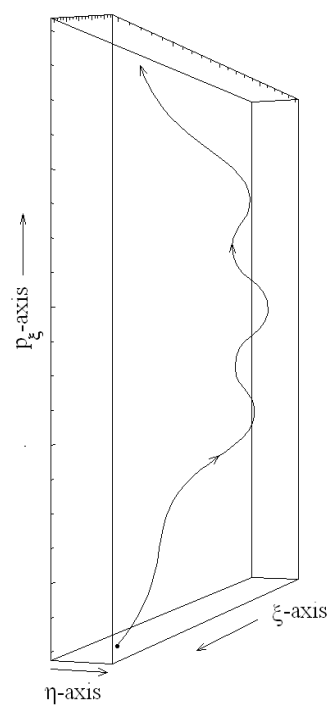

(b)

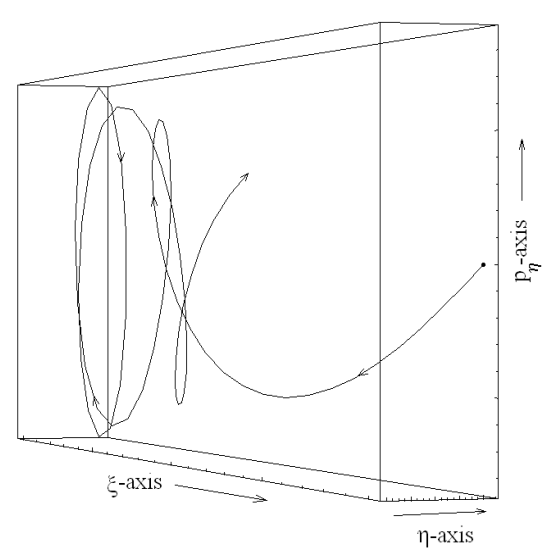

Figure 12: Three-dimensional views of ray D of Figure 10: (a) in $\left(\xi, \eta, p_{\xi}\right)$ subspace and (b) in $\left(\xi, \eta, p_{\eta}\right)$ subspace. 

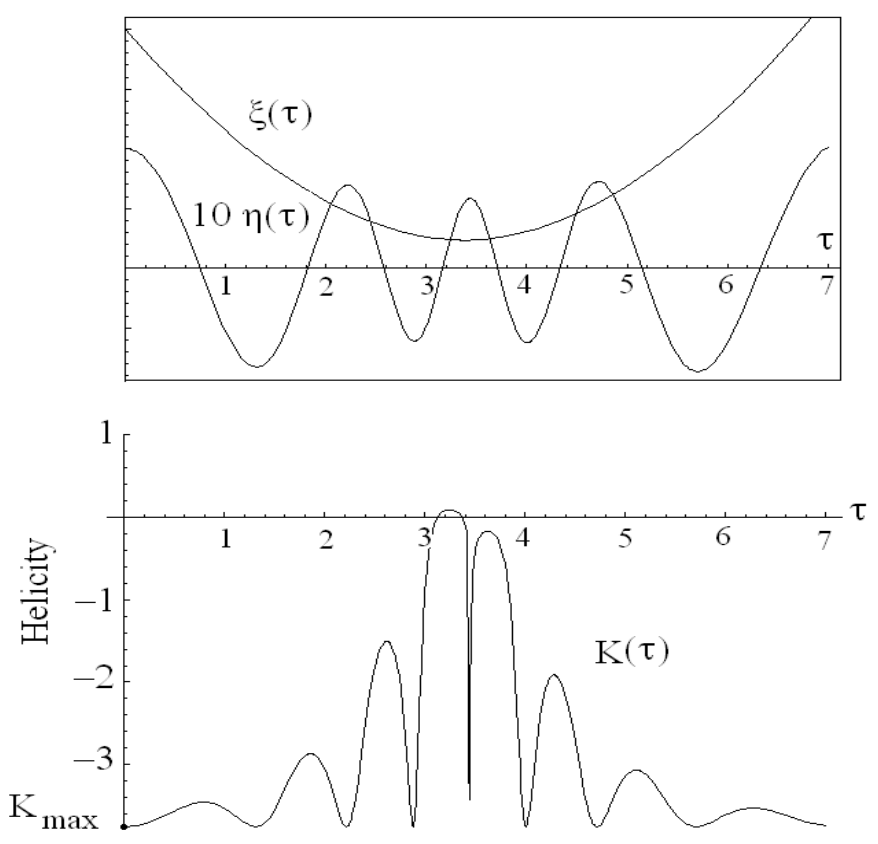

Figure 13: Plots of helicity $K, \xi$, and $\eta$ (multiplied by a factor 10) for ray D of Figure 10 as functions of the orbit parameter $\tau$. Here, for $b=0.25$, we have $K_{\max }=b-b^{-1}=-3.75$. We note that the helicity reaches its maximum (negative) value when the oscillatory $\eta$ motion reaches its turning points and, thus, tunneling through the evanescent regions is maximally affected by the helicity. 
exponentiation rate $\gamma_{\xi}$ and, for finite $\left(\eta, p_{\eta}\right)$, the $\eta$-action

$$
J_{\eta} \equiv \frac{1}{2 \omega_{\eta}}\left(p_{\eta}^{2}+\omega_{\eta}^{2} \eta^{2}\right)
$$

is, therefore, approximately an adiabatic invariant. Hence, the ray Hamiltonian for the $\xi$-motion is

$$
H^{\prime}\left(\xi, p_{\xi} ; J_{\eta}\right)=\frac{1}{2} p_{\xi}^{2}+V\left(\xi ; J_{\eta}\right)
$$

where the effective potential is

$$
V\left(\xi ; J_{\eta}\right) \equiv-\frac{1}{2} b \xi+J_{\eta} \omega_{\eta}(\xi), \text { with } \omega_{\eta}(\xi)=(b \xi)^{-\frac{1}{2}}
$$

Thus, the $\eta$-oscillation acts as a repulsive potential for the $\xi$-motion (analogous to magnetic mirroring of a gyrating charged particle). Note that the Hamiltonian (25) is a ray guidingcenter Hamiltonian, averaged over $\eta$-oscillations, and that the ray guiding-center $\xi$-motion, defined by the solution of the ray guiding-center Hamilton's equations $\dot{\xi}=p_{\xi}$ and $\dot{p}_{\xi}=$ $b / 2+J_{\eta} /\left(2 \sqrt{b \xi^{3}}\right)$, oscillates about the exact $\xi$-motion (see Figure 14 ).

Since $H^{\prime}\left(\xi, p_{\xi} ; J_{\eta}\right)=0$, the turning point $\xi_{\text {tp }}$ (where $p_{\xi}=0$; see Figure $11(\mathrm{a})$ ) is determined from $V\left(\xi ; J_{\eta}\right)=0$ :

$$
\xi_{\text {tp }}\left(J_{\eta}\right)=\left(2 J_{\eta}\right)^{\frac{2}{3}} \cot \frac{\alpha}{2}
$$

Expressing the $\eta$-action (24) in terms of the initial conditions $\left(\xi_{0}, \eta_{0}, p_{\eta 0}=0\right)$, we have

$$
J_{\eta}=\frac{1}{2} \eta_{0}^{2}\left(b \xi_{0}\right)^{-\frac{1}{2}}
$$

and we obtain

$$
\frac{\xi_{\mathrm{tp}}}{\xi_{0}}=\left(\frac{\left|\eta_{0}\right|}{b \xi_{0}}\right)^{\frac{4}{3}} .
$$

This equation shows how rays with larger $\left|\eta_{0}\right|$ have turning points farther from the apex of the wedge.

Note that, for fixed values of $b$ and $\xi_{0}$, the $\eta$-action scales as $J_{\eta} \sim \eta_{0}^{2}$ and the turning point scales as $\xi_{\text {tp }} \sim\left|\eta_{0}\right|^{4 / 3} \sim J_{\eta}^{2 / 3}$. In Figure 10, we see that orbits with small values of $\eta_{0}$ have turning points closer to the apex of the wedge, since they experience a weaker retarding force. For fixed $\left(\xi_{0}, \eta_{0}\right)$ and $p_{\eta 0}=0$, the $\eta$-action $(24)$ scales as $J_{\eta} \sim b^{-1 / 2}$ and the turning point scales as $\xi_{\mathrm{tp}} \sim b^{-4 / 3}$; hence, wedges with large $b$ (for fixed $\xi_{0}$ and $\eta_{0}$ ) have turning points closer to their apex.

\section{Summary of the Symmetric-Wedge Model}

In this Section, we introduced a symmetric-wedge model as a result of the appearance of the two-dimensional six-sector topology in the vicinity of the intersection points found 


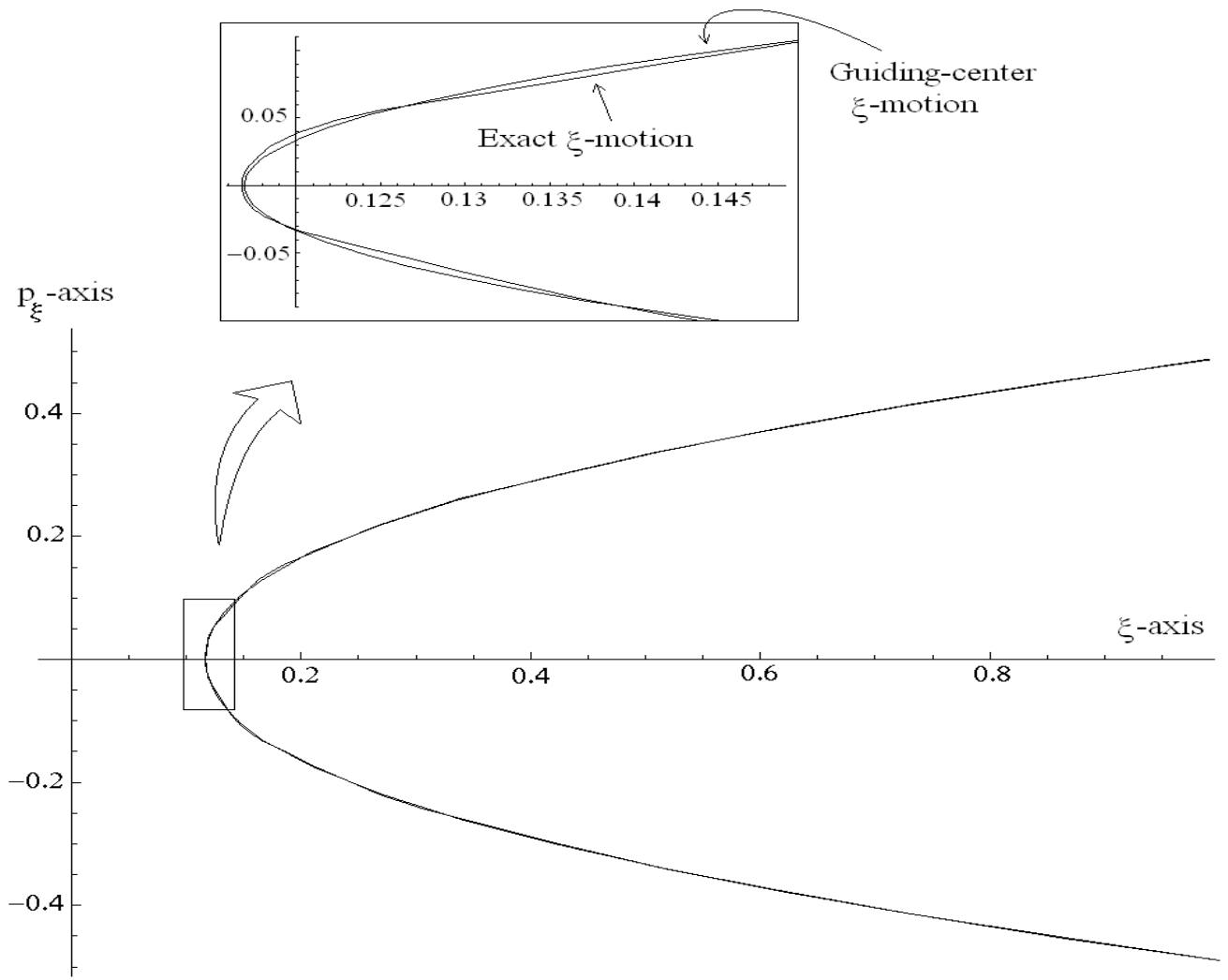

Figure 14: Plots of the exact and guiding-center $\xi$-motions on the $\left(\xi, p_{\xi}\right)$ plane for ray D of Figure 10. The difference between the two types of motion is barely visible except near the turning point (see inset). 
in Figures 4 and 5. We then constructed a simple wedge Hamiltonian (20) and studied the solutions of the Hamiltonian dynamics in the propagating region I, in which incident and reflected magnetosonic waves propagate, by solving numerically the Hamilton's equations (22)-(23). The results are shown graphically in Figures 10, 11, and 12, which attest to the complexity of the helical rays generically found in region I.

\section{SUMMARY AND CONCLUSIONS}

In this paper we have shown that nonzero helicity arises generically in the cold-plasma model wherever the density and magnetic field gradients are not parallel. We then examined the behavior of rays in a simple two-dimensional tokamak model with a D-T plasma (ignoring poloidal field effects). Computing the dispersion surface (i.e., the zero locus of the determinant of the dispersion matrix in the ray phase space), we identified special points in physical space where two branches of the dispersion manifold touch. The essential features of the ray dynamics near these regions can be captured by a very simple model, which we call the "symmetric-wedge" model. Preliminary studies of the effects of adding a nonzero poloidal magnetic field suggest that the shape of the surfaces in phase space are smoothly deformed, except perhaps right at the point where the two surfaces touch. This is currently under investigation. While this result is unsurprising from a mathematical point of view, it does lead to important new physical effects (e.g., that part of the dispersion surface we identify as being associated with the ion-hybrid wave is no longer vertical, implying that this disturbance now propagates in $\mathbf{x}$-space as well as $\mathbf{k}$-space). This will be discussed in a separate paper devoted to this topic. In addition, as it currently stands, it is unclear how to extend the numerical ray tracing algorithm of Ref. [8] to allow for helical rays. Preferably there would be one algorithm that could deal with arbitrary helicity cases (including zero helicity).

While of intrinsic scientific interest, it is unclear how significant these helical regions will turn out to be for tokamak research. It is possible that they will play only a minor role in the radio-frequency (RF) response of the tokamak plasma. This is because they are localized in the ray phase space, and it is likely that only a small fraction of rays will ever encounter these regions before they have lost their initial energy due to damping on the plasma or more familiar conversion processes. However, the transmission of energy from one branch of the dispersion surface to the other will be significant in these regions; hence it is possible that they could play a significant role in certain parameter ranges. For example, by exploiting the parameter dependence and strong spatial localization of helical regions, it could be arranged so that a significant fraction of the rays launched from an antenna will enter the helical region. This effect could potentially be used to achieve finer 
control over RF heating or current drive profiles.

\section{Acknowledgments}

We wish to thank R.G. Littlejohn and André Jaun for useful discussions. This work was supported by the U.S. Dept of Energy under Contract No. DE-AC03-76SFOO098. E.R. Tracy acknowledges support by the National Science Foundation under Grant No. 0317256 . 


\section{References}

[1] E.R. Tracy, A.N. Kaufman, and A.J. Brizard, Phys. Plasmas 10, 2147 (2003).

[2] D.G. Swanson, Theory of Mode Conversion and Tunneling in Inhomogeneous Plasmas (John Wiley \& Sons, 1998).

[3] R.A. Cairns and C.N. Lashmore-Davies, Phys. Fluids 26, 1268 (1983).

[4] V. Fuchs, A. Bers, and L. Harten, Phys. Fluids 28, 177 (1985)

[5] L. Friedland and A.N. Kaufman, Phys. Fluids 30, 3050 (1987).

[6] R.G. Littlejohn, private communication.

[7] E.R. Tracy and A.N. Kaufman, Phys. Rev. E 48, 2196 (1993).

[8] E.R. Tracy, A.N. Kaufman, and A. Jaun, Phys. Lett. A 290, 309 (2001).

[9] E.R. Tracy and A.N. Kaufman, Phys. Rev. Lett. 91, 130402 (2003).

[10] R.G. Littlejohn and W.G. Flynn, Chaos 2, 149 (1992).

[11] R.G. Littlejohn and W.G. Flynn, Phys. Rev. Lett. 70, 1799 (1993).

[12] W.G. Flynn and R.G. Littlejohn, Ann. Phys. (N.Y.), 234, 334 (1994).

[13] E.R. Tracy and A.N. Kaufman, in preparation.

[14] T.H. Stix, Waves in Plasmas (American Institute of Physics, New York, 1992).

[15] F.W. Perkins, Nuc. Fusion 17, 1197 (1977).

[16] Y. Colin de Verdière, Ann. Inst. Fourier 53, 1023 (2003).

[17] P.J. Braam and J.J. Duistermaat, Indag. Math. 4, 407 (1993). 